\title{
Altas habilidades/superdotação: o que dizem as pesquisas sobre estas crianças invisíveis?
}

\author{
Bárbara Amaral Martins \\ Universidade Federal do Mato Grosso do Sul - Campus do Pantanal - Corumbá - MS - Brasil \\ Ketilin Mayra Pedro \\ Universidade Estadual Paulista - Marilia - SP - Brasil \\ Clarissa Marques Maria Ogeda \\ Universidade Estadual Paulista - Marília - SP - Brasil
}

\begin{abstract}
Resumo
Este estudo tem por objetivo analisar as teses e dissertações brasileiras, no período de 2005 a 2014, que abordam a temática da identificação de estudantes com altas habilidades/superdotação. Realizamos um levantamento junto ao Banco de Teses e Dissertações da Coordenação de Aperfeiçoamento Pessoal de Nível Superior, à Biblioteca Brasileira de Teses e Dissertações, à Plataforma Sucupira e aos principais Programas de Pós-Graduação com produção na área da Educação Especial no Brasil. Os descritores utilizados foram Identificação, Altas Habilidades, Superdotação, Precocidade, Talento e Dotação. Ao analisar os resultados constatamos que $20 \%$ do total de publicações abordam a temática da identificação, sendo 2012 o ano com maior número de publicações. Em relação ao local e tipo de publicações, destacam-se as dissertações e a Universidade Federal de São Carlos. Quanto à categorização das pesquisas, destacamos a identificação de estudantes em contextos específicos, públicos específicos e elaboração de instrumentos de identificação.
\end{abstract}

Palavras-chave: Superdotados; pesquisa científica; identificação.

\section{High skills / giftedness: what do the research on these invisible children say?}

\begin{abstract}
This study aims to analyze Brazilian theses and dissertations, from 2005 to 2014, which deal with the identification of students with high skills / giftedness. We conducted a survey with the Bank of Theses and Dissertations of the Coordination of Personal Improvement of Higher Education, the Brazilian Library of Thesis and Dissertations, the Platform Sucupira and the main Graduate Programs with production in the area of Special Education in Brazil. The descriptors used were Identification, High Abilities, Giftedness, Precocity, Talent and Endowment. When analyzing the results we found that $20 \%$ of all publications deal with identification, with 2012 being the year with the highest number of publications. Regarding the place and type of publications, the dissertations and the Federal University of São Carlos stand out. Regarding the categorization of research, we highlight the identification of students in specific contexts, specific publics and the elaboration of identification instruments.
\end{abstract}

Keywords: Gifted; scientific research; identification.

\section{Altas habilidades/superdotación: ¿lo qué dicen las investigaciones sobre estos niños invisibles?}

\section{Resumen}

Este estudio tiene por objetivo analizar las tesis y tesinas brasileñas, en el período de 2005 a 2014, que abordan la temática de la identificación de estudiantes con altas habilidades/superdotación. Realizamos un levantamiento junto al Banco de Tesis y Tesinas de la Coordinación de Mejora Personal de Nivel Superior, a la Biblioteca Brasileña de Tesis y Tesinas, a la Plataforma Sucupira y a los principales Programas de Postgrado con producción en el área de la Educación Especial en Brasil. Los descriptores utilizados fueron Identificación, Altas Habilidades, Superdotación, Precocidad, Talento y Dotación. Al analizar los resultados constatamos que el 20\% del total de publicaciones abordan la temática de la identificación, siendo 2012 el año con número más elevado de publicaciones. En relación al sitio y tipo de publicaciones, se destacan las tesinas y la Universidad Federal de São Carlos. Cuanto a la categorización de las investigaciones, destacamos la identificación de estudiantes en contextos específicos, públicos específicos y elaboración de instrumentos de identificación.

Palabras clave: Superdotados; investigación científica; identificación. 


\section{Introdução}

A Educação Especial é um campo que traz para as discussões educacionais "os aspectos mais frágeis da Educação Brasileira” (Anache, 2009, p. 123), e, como pesquisadores da área, acreditamos que todos os estudantes têm direito a uma educação de qualidade, independentemente das suas condições físicas, sociais e intelectuais.

A Lei de Diretrizes e Bases da Educação Nacional (LDB), Nº. 9.394/96 dispõe em seu artigo $58^{\circ}$ que a Educação Especial é "a modalidade de educação escolar oferecida preferencialmente na rede regular de ensino, para educandos com deficiência, transtornos globais do desenvolvimento e altas habilidades ou superdotação"' (Lei n 9.394, 1996, p. 33 ), garantindo em seu artigo $59^{\circ}$, inciso I, que os sistemas de ensino assegurarão a esses alunos, "currículos, métodos, técnicas, recursos educativos e organização específicos, para atender às suas necessidades" (Lei n 9.394, 1996, p. 34).

Dentre os estudantes que compõe o público-alvo da Educação Especial, os alunos com AH/SD não são aqueles que mais recebem atenção por parte da mídia, das políticas governamentais para a educação ou mesmo da comunidade acadêmica, contudo, nota-se uma tendência de crescimento nos estudos relacionados a essa temática (Chacon \& Martins, 2014).

No entanto, quando se trata de pesquisa científica, não basta haver somente crescimento quantitativo, é necessário o acompanhamento de avanço qualitativo, o que exige um olhar sobre o corpus da pesquisa na área. De acordo com Omote (2014, p. 21),

[...] há hoje um farto volume de publicações em periódicos e anais de eventos tratando de uma ampla diversidade de problemas que vêm a propósito na compreensão e equacionamento de questões implicadas na Educação Inclusiva e de seus correlatos. Para um avanço qualitativo expressivo pode estar faltando uma ampla revisão e sistematização dos resultados encontrados, e principalmente um vigoroso esforço para uma análise crítica e incorporação desses achados no corpo de conhecimentos já existente para a construção de referenciais teóricos sólidos [...]

Nesse sentido, reconhecemos a importância do empreendimento de análises acerca do conhecimento produzido na área das $A H / S D$, em especial ao que se refere à identificação desses indivíduos, pois "ainda não se tem certeza de quem são e onde estão essas pessoas com AH/ SD", o que instala uma problemática que só poderá ser resolvida por meio da própria pesquisa (Freitas, 2014, p. 132).

Estudos acerca da identificação são relevantes na medida em que a atenção educacional destinada a esses estudantes depende, inicialmente, de tal processo, o qual deve realizar-se o quanto antes, desde a educação infantil, dada a necessidade de promover atenção educacional

1 Ao longo deste artigo utilizaremos a nomenclatura altas habilidades/superdotação (AH/SD), em consonância com o Conselho Brasileiro para Superdotação (ConBraSD). imediata, a fim de evitar problemas de ajustamento, falta de interesse ou baixo desempenho (Resolução $n^{\circ} 2,2001$; Guimarães \& Ourofino, 2007).

Apesar de ser imprescindível, ainda estamos distantes de identificar adequadamente e em grande escala aqueles que possuem habilidades superiores e requerem adequações educacionais (Martins, 2013). Por essa razão, é necessário olhar para os achados das pesquisas acadêmicas com vistas a compreender quais são as suas contribuições e que caminhos podemos trilhar para avançar na produção de conhecimento sobre o processo de identificação.

Assim, este estudo tem por objetivo analisar as teses e dissertações brasileiras defendidas no período de 2005 a 2014, que abordam a temática da identificação de estudantes com AH/SD.

\section{Método}

Realizamos um levantamento junto ao Banco de Teses e Dissertações da Coordenação de Aperfeiçoamento Pessoal de Nível Superior (CAPES), à Biblioteca Brasileira de Teses e Dissertações, à Plataforma Sucupira e aos principais Programas de Pós-Graduação com produção na área da Educação Especial no Brasil. Destaca-se que alguns dos trabalhos se repetiram entre as fontes utilizadas, gerando a necessidade de realizar uma comparação entre os resultados de todos os portais para que fossem eliminadas as duplicidades.

O intervalo temporal utilizado compreendeu um período de 10 anos (2005 a 2014) e os descritores utilizados foram Identificação, Altas Habilidades, Superdotação, Precocidade, Talento e Dotação, que deveriam estar presentes no título, resumo ou palavras-chave. A variedade de descritores utilizados no levantamento bibliográfico se deve à ausência de consenso em relação à terminologia utilizada na área, havendo diferenciações conforme o referencial teórico utilizado; dessa maneira, optamos por utilizar todas as terminologias vigentes em âmbito nacional.

A análise dos dados caracteriza-se como qualitativa. Aqueles passíveis de quantificação, como período de publicação, universidade de origem e outros, foram tabulados e analisados descritivamente, sendo que os não quantificáveis foram identificados por meio das leituras das produções encontradas.

Ressaltamos que as teses e dissertações analisadas não representam a totalidade de produções indexadas nas bases de dados utilizadas, sendo objeto de estudos apenas aquelas que versavam sobre a temática das altas habilidades/superdotação.

\section{Resultados e Discussões}

Por meio do levantamento das teses e dissertações sobre AH/SD defendidas no período de 2005 a 2014, encontramos um total de 91 produções, sendo que 18 delas $(20 \%)$ 
têm como objeto de investigação a identificação, como mostra a Figura 1.

घ Identificação = Demais Temáticas

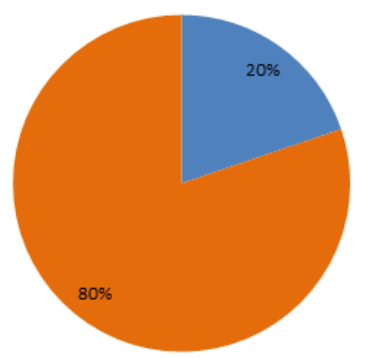

Figura 1. A temática da identificação no universo das produções acadêmicas em altas habilidades/superdotação.

A Figura 1 evidencia que há uma parcela expressiva das produções em AH/SD que se voltam para a temática da identificação. Contudo, embora os trabalhos a respeito da identificação estejam entre os mais numerosos, o número de estudantes registrados como AH/SD ainda é inexpressivo. De acordo com Freitas (2014), estima-se que 5\% da população estudantil brasileira têm AH/SD, o equivalente a 2,5 milhões de estudantes; porém, apenas 11.025 estudantes $(0,44 \%)$ encontram-se oficialmente identificados.

A figura a seguir apresenta a distribuição dessas pesquisas segundo o ano de publicação.

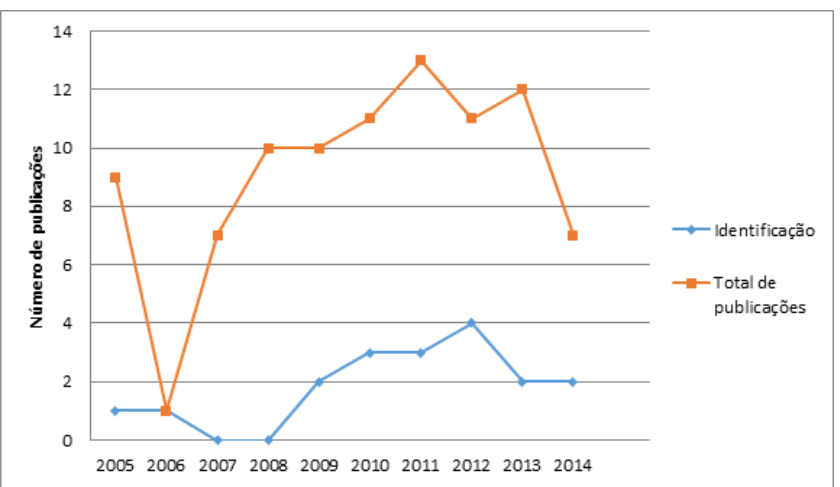

Figura 2. Número de publicações no período de 2005 a 2014.

Observando a linha dos trabalhos sobre identificação, nota-se que no período de 2005 a 2008, a referida temática foi pouco investigada no âmbito da pesquisa acadêmica; porém, houve crescimento no período de 2009 a 2012 (acompanhando o aumento no total de produções), seguido de decréscimo em 2013. Destaca-se que no ano de 2006 houve a defesa de um único estudo sobre $A H / S D$, justamente a respeito da identificação. Em 2008, quando se iniciou o período de maior produtividade no total de publicações, nenhum estudo abordou a temática da identificação. Vale ressaltar que os anos de 2005 e 2007 foram importantes para a área, sendo que no primeiro foram implantados os Núcleos de Atividades de Altas Habilidades/Superdotação (NAAH/S), com o objetivo de oferecer atendimento educacional especializado para esses estudantes, bem como orientar suas respectivas famílias e professores, enquanto que em 2007 foi elaborada a Política Nacional de Educação na Perspectiva da Educação Inclusiva, publicada no ano seguinte, que impulsionou o debate na área e também pode ter proporcionado um maior interesse na realização das pesquisas.

A Figura 3 demostra que as produções acadêmicas foram desenvolvidas, principalmente em nível de mestrado, convergindo com a produção total na área das AH/SD.

$$
\text { [ Identificação = Total de produções }
$$

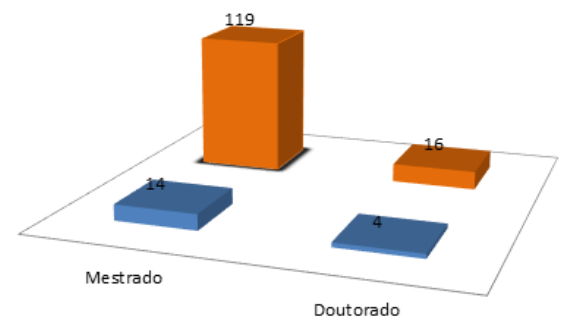

Figura 3. Distribuição das pesquisas segundo o nível de pósgraduação em que foram produzidas.

A desproporcionalidade entre estudos realizados em nível de mestrado e doutorado é completamente compreensível na medida em que a área da Educação é a que mais tem desenvolvido pesquisas acerca das AH/SD (Chacon \& Martins, 2014) e, nesta área, o país tem mais cursos de pós-graduação em mestrado acadêmico (126) do que em doutorado (66), além de serem estes mais longos que aqueles, o que ocasiona uma produtividade anual quantitativamente menor.

Na Figura 4 são apresentadas as universidades nas quais os estudos sobre identificação foram desenvolvidos.

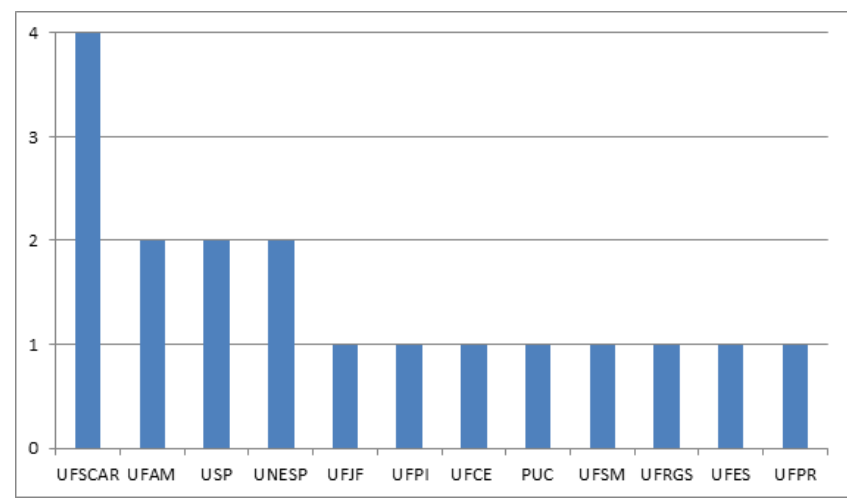

Figura 4. Distribuição das pesquisas segundo o local de publicação.

Entre as 12 universidades em que pesquisadores se dedicaram a investigar a identificação destaca-se a Universidade Federal de São Carlos (UFSCAR), com quatro estudos, seguida da Universidade Federal do Amazonas (UFAM), Universidade de São Paulo (USP) e Universidade Estadual Paulista "Júlio de Mesquita Filho" (UNESP), todas 
Tabela 1. Categorização das pesquisas sobre identificação de alunos com altas habilidades/superdotação, no período de 2005 a 2014.

\begin{tabular}{lc}
\hline Categoria & Número de pesquisas \\
\hline Identificação de alunos com AH/SD em contextos específicos & 4 \\
\hline Identificação de AH/SD em públicos específicos & 4 \\
\hline Elaboração de novas propostas/instrumentos & 4 \\
\hline Análise de concepções e/ou percepções de pessoas envolvidas & 3 \\
\hline Capacitação de professores para a identificação & 2 \\
\hline Análise de documentos legais & 1 \\
\hline TOTAL & 18 \\
\hline
\end{tabular}

Fonte: Elaboração própria.

com três. Com apenas uma produção elencamos: Universidade Federal do Piauí, Universidade Federal do Ceará, Pontifícia Universidade Católica (PUC-Campinas), Universidade Federal de Santa Maria, Universidade Federal do Rio Grande do Sul, Universidade Federal do Espírito Santo e Universidade Federal do Paraná.

Identificar alunos com AH/SD é uma atitude imprescindível na medida em que, quando não são reconhecidos e estimulados, correm o risco de se adaptarem ao contexto rotineiro da sala de aula, deixando de desenvolver suas habilidades e, até mesmo, tornando-se desinteressados e frustrados (Freeman \& Guenther, 2000). Preocupados com essa questão, pesquisadores têm desenvolvido estudos distintos, mas com a mesma finalidade: contribuir com o processo de identificação. A seguir, apresentamos a categorização das pesquisas localizadas:

A Tabela 1 nos mostra que há um equilíbrio entre as pesquisas que se dedicaram à identificação de alunos com AH/SD em contextos específicos, identificação de $\mathrm{AH} /$ $S D$ em determinados públicos e elaboração de novas propostas/instrumentos, todas com quatro estudos. Logo em seguida, com três pesquisas, temos a análise de concepções e/ou percepções de pessoas envolvidas, capacitação de professores para a identificação, com duas, e análise de documentos legais, com uma pesquisa.

Com relação à categoria identificação de alunos com AH/SD em contextos especificos, Fonseca (2010) se propôs a identificar alunos com AH/SD na área acadêmica em uma escola particular, na cidade de Teresina/Piauí. A partir da identificação de seis estudantes, caracterizou o perfil dos mesmos, identificou as relações estabelecidas com os colegas, professores e família e o desempenho escolar que apresentam. Marques (2011) realizou um levantamento de alunos com indicadores de AH/SD com a participação de 76 professores do Ensino Fundamental I de um município paulista, a partir de um formulário de indicadores sobre AH/SD. Pereira (2010) realizou uma comparação entre as perspectivas de Guenther e Renzulli implementando um Programa de Identificação de Estudantes Talentosos em um colégio de uma universidade pública. Participaram da investigação 21 educadores e 393 alunos do quinto ao oitavo ano do ensino fundamental, bem como os pais desses estudantes, sendo que os instrumentos utilizados foram: testes de inteligência, escalas e questionários e roteiro de observação para professores.

Marques (2013) objetivou reconhecer sinais de precocidade nas crianças a partir das informações de pais e professores. A pesquisa foi realizada com 46 alunos (3 e 4 anos), quatro pais e oito professores de uma escola municipal de educação infantil do interior do estado de São Paulo, tendo como instrumentos um questionário socioeconômico respondido pelos pais, questionário de indicadores de precocidade para os pais e professores e ficha de caracterização do professor participante.

$\mathrm{Na}$ categoria identificação de $A H / S D$ em públicos específicos, temos o trabalho de Cardoso (2009), que identificou quatro adolescentes em situação de rua com potencial para AH/SD participantes do Programa Social "Criança Urgente", desenvolvido por um município do estado do Amazonas, delineando suas características e potencialidades; o de Negrini (2009), que adaptou e implementou estratégias de identificação de alunos com AH/SD em uma escola de surdos, averiguando os efeitos deste processo para a instituição e analisando as percepções dos envolvidos (alunos, professores regentes e auxiliares) sobre alunos com AH/SD e identificação; o de Ragni (2012), que realizou uma pesquisa exploratória, do tipo estudo de caso, com o objetivo de identificar o talento em alunos com perdas auditivas de diferentes graus, com a participação de 52 alunos, matriculados no ensino fundamental e médio de duas escolas da rede pública, uma localizada no interior do estado e outra na capital; e o estudo de Ivo (2012), que pesquisou a criatividade na identificação e seleção para um determinado projeto de crianças e adolescentes com AH/SD, utilizando uma amostra de dez jovens em situação de carência socioeconômica e questionando por que o número de vagas para um determinado projeto não era preenchido e se a causa era a valorização apenas do tipo de superdotação acadêmica em detrimento da criativo-produtiva. 
Quanto à categoria elaboração de novas propostas/ instrumentos, Vieira (2005) analisou a identificação de $\mathrm{AH} /$ SD em crianças de quatro a seis anos de idade, sistematizando um procedimento que considera os aspectos cognitivos, afetivos, psicomotores e sociais, em um centro de identificação de AH/SD. Farias (2012) elaborou uma escala de nomeação docente para alunos dotados e talentosos e buscou evidências de precisão da consistência interna. O instrumento ENDI-p foi testado, comprovando-se que está adequado para utilização. Christofoletti (2012) realizou uma pesquisa quantitativa, transversal e correlacional, desenvolvendo um método de interpretação probabilística para identificar alunos talentosos a partir do modelo dos três anéis de Renzulli, o chamado Método das Hélices. E Martins (2013) identificou comportamentos e indicadores de precocidade, situações em sala de aula que favorecem ou desfavorecem a manifestação da precocidade e verificou se determinados alunos apresentam as características de AH/SD, elaborando, por fim, um manual de orientação para que professores identifiquem alunos com precocidade.

No que diz respeito à análise de concepções e/ou percepções de pessoas envolvidas, Lima (2011) investigou se os professores universitários reconhecem os alunos com $\mathrm{AH} / \mathrm{SD}$, como o fazem e as estratégias metodológicas que adotam para a inclusão educacional destes, entrevistando nove participantes. Oliveira (2014) investigou como os pais ou responsáveis identificam a precocidade, quais expectativas têm sobre essas crianças e como percebem as alterações na dinâmica familiar devido à precocidade. Como instrumentos foram utilizados dois questionários respondidos por 19 participantes de um programa que oferece atenção a estudantes com esse perfil. Cruz (2014) realizou uma análise das concepções de AH/SD sob a ótica dos professores de educação especial que atuam na área por meio de suas narrativas, e também a influência dessas concepções na identificação desses estudantes, utilizando um grupo focal como técnica de coleta.

$\mathrm{Na}$ categoria capacitação de professores para a identificação temos dois estudos, sendo um realizado por Martins (2006), que propôs um processo de identificação de alunos com potencial para AH/SD no Ensino Fundamental II em uma escola adventista, com indicação por pares, professores e auto-nomeação, analisando a diferença existente entre a indicação realizada por parte de professores antes e após capacitação e localizando características de problemas, dificuldades de aprendizagem ou sub-desempenho nos alunos indicados. O outro, de Araújo (2011), desenvolveu uma proposta de atuação para professores do Atendimento Educacional Especializado (AEE) para identificação e encaminhamento de alunos com características de AH/SD, por meio de capacitação em serviço. Para tal comparou as concepções dos envolvidos acerca dos alunos e também os indicadores de AH/SD assinalados pelo professor. Utilizou uma amostra de 865 alunos e 87 profissionais da educação.

$\mathrm{Na}$ categoria análise de documentos legais, temos a pesquisa de Souza (2011), que objetivou identificar e caracterizar os alunos com deficiência, transtornos globais do desenvolvimento e AH/SD inclusos nas salas regulares da rede pública estadual por meio da consulta de documentos oficiais disponibilizados pela Diretoria Regional de Ensino e do MEC, analisando as matrículas ocorridas no período de 2005 a 2009.

Todos os estudos anteriormente mencionados são de vasta relevância para a efetivação da proposta inclusiva para esse alunado, e nesse cenário, destacamos o pequeno número de trabalhos que focalizaram a capacitação de professores, pois a urgência de formação docente para atuar com alunos com AH/SD já foi constatada por Martins e Alencar (2011), em virtude das informações reduzidas e, por vezes, errôneas que educadores apresentam. Ressalta-se ainda, a necessidade dos estudos de natureza documental, em especial os que analisam a situação do número de matrículas de alunos com AH/SD, como realizado por Souza (2011), em vista do grande desafio que é tirá-los da invisibilidade. Segundo Pérez e Freitas (2011), o EDUDATABRASIL, sistema de estatísticas educacionais do Instituto Nacional de Estudos e Pesquisas Educacionais Anísio Teixeira (INEP), traz dados de matrículas de alunos com AH/SD somente nos anos de 1999 a 2002, onde são registradas respectivamente, 468, 454, 692, 625 matrículas, em todo o território nacional, enquanto as estimativas mais conservadoras (3,5 a $5 \%$ da população) previam uma média de 2.250.182, 2.240.499, 2.330.052 e 2.348.798 de matrículas para os mesmos anos. Apesar de os números apresentados pelo INEP referentes aos anos de 2007 e 2008 serem consideravelmente mais favoráveis (2.982 e 3.676, respectivamente), mostram o quão distantes ainda estamos tanto da oferta de atenção educacional adequada quanto da própria identificação desses educandos.

\section{Considerações Finais}

Ao analisar as teses e dissertações defendidas no intervalo temporal de 2005 a 2014, relacionadas à temática da identificação de estudantes com AH/SD, constatamos que $20 \%$ do total de publicações abordam a temática elencada como foco para esta pesquisa, sendo 2012 o ano com maior número de publicações. Em relação ao local e tipo de publicações destacam-se as dissertações e a Universidade Federal de São Carlos, que teve sua primeira dissertação defendida sobre o tema no ano de 2010.

Em relação à categorização das pesquisas sobre a identificação de alunos com AH/SD, percebemos que as temáticas mais exploradas estão relacionadas com a identificação de alunos tanto em contextos públicos e privados, como também a elaboração de novas propostas e instrumentos.

É importante apontar que além de a produção acadêmica ser incipiente na área das AH/SD e pouco expressiva em relação à temática da identificação, carecemos também de instrumentos de avaliação nacional sistematizados que sejam validados e possam ser utilizados em grande escala. Embora os documentos oficiais apontem diretrizes para 
a identificação desses estudantes e algumas produções tenham como objetivo fortalecer esta área, sentimos que muitas vezes falta cientificidade e aplicação em larga escala desses instrumentos para que eles possam trazer dados confiáveis sobre identificação. Tais carências fazem com que os estudantes AH/SD sejam invisíveis dentro do contexto escolar, sendo que muitas vezes seu potencial passa despercebido e/ou não é valorizado.

Além disso, existe a recomendação de que os profissionais realizem uma avaliação holística dos estudantes, em que o potencial psicológico e acadêmico seja valorizado, considerando também a realidade cultural em que o estudante está inserido, bem como as informações de seus familiares e professores.

Diante do exposto, percebemos a necessidade de aprofundar os estudos nessa área e também realizar pesquisas que possam trazer novos apontamentos e instrumentos para a identificação deste alunado.

\section{Referências}

Anache, A. A. (2009). A epistemologia qualitativa: contribuições para a pesquisa em educação especial. InterMeio: Revista do Programa de Pós-Graduação em Educação, 15 (30), 123-141.

Araújo, M. R. (2011). Identificação e encaminhamento de alunos com indicadores de altas habilidades/superdotação na escola pública do município de fortaleza: proposta para a atuação de professores do atendimento educacional especializado. Dissertação de Mestrado em Educação Brasileira, Faculdade de Educação, Universidade Federal do Ceará, Fortaleza. Resuperado: 02 jul. 2015. Disponível:http://www.repositorio.ufc.br/bitstream/ riufc/3655/1/2011_DIS_MRARAUJO.pdf.

Biblioteca Digital Brasileira de Teses e Dissertações [BDTD] (s.d.). Banco de Teses e Dissertações. Recuperado: 11 dez. 2014. Disponível: http://bdtd.ibict.br/.

Coordenação de Aperfeiçoamento de Pessoal de Nível Superior [CAPES] (s.d.) Banco de teses. Recuperado: 20 nov. 2014. Disponível: http://www.capes.gov.br/capes/portal/conteúdo/10/ banco_teses.htm.

Coordenação de Aperfeiçoamento de Pessoal de Nível Superior [CAPES] (s.d.). Relação dos cursos recomendados e reconhecidos. Recuperado: 27 jun. 2015. Disponível:http://conteudoweb.capes. gov.br/conteudoweb/ProjetoRelacaoCursosServlet?acao=pesquis arAreaAvpesquis\#.

Cardoso, A. O. G. (2009). Identificando adolescentes em situação de rua com potencial para altas habilidades/superdotação. Dissertação de Mestrado em Educação, Universidade Federal do Amazonas, Manaus. Recuperado: 02 jul. 2015. Disponível: http:// www.dominiopublico.gov.br/download/texto/cp150234.pdf .

Chacon, M. C. M. \& Martins, B. A. A produção acadêmico-científica do
Brasil na área das altas habilidades/superdotação no período de 1987 a 2011. Revista Educação Especial, 27 (49), 353-372.

Christofoletti, R. A. (2012). Proposta de aplicação do método das hélices na identificação de estudantes talentosos. Dissertação de Mestrado em Educação Especial, Universidade Federal de São Carlos, São Carlos. Recuperado: 25 jun. 2015. Disponível: http:// www.bdtd.ufscar.br/htdocs/tedeSimplificado//tde_busca/arquivo. php?codArquivo $=5095$.

Cruz, C. (2014). Serão as altas habilidades/superdotação invisíveis? Tese de Doutorado em Educação, Universidade Federal do Espírito Santo, Vitória. Recuperado: 02 jul. 2015. Disponível: https://sucupira.capes.gov.br/sucupira/public/consultas/coleta/ trabalhoConclusao/viewTrabalhoConclusao.jsf?popup=true\&id_ trabalho=1930700.

Farias, E. S. (2012). Elaboração de instrumento para identificação de alunos intelectualmente dotados por professores: estudo exploratório. Tese de Doutorado em Psicologia, Pontifícia Universidade Católica de Campinas, Campinas. Recuperado: 02 jul. 2015. Disponível: http://www.bibliotecadigital.puc-campinas. edu.br/tde_busca/arquivo.php?codArquivo=765 .

Fonseca, D. F. (2010). A identificação de alunos com altas habilidades/ superdotação em uma escola da rede particular de ensino de Teresina-Piauí. Dissertação de Mestrado em Educação, Centro de Ciências da Educação, Universidade Federal do Piauí. Recuperado: 25 jun. 2015. Disponível: http://www.ufpi.br/subsiteFiles/ppged/ arquivos/files/dissertacao/2010/DANIELLE.pdf.

Freeman, J. \& Guenther, Z. C. (2000). Educando os mais capazes: idéias e ações comprovadas. São Paulo: EPU.

Freitas, S. N. (2014). Altas Habilidades/ Superdotação em Pesquisa: Um olhar dirigido. Em S. Omote, A. A. S. Oliveira, \& M. C. M. Chacon (Orgs.), Ciência e Conhecimento em Educação Especial. São Carlos: Marquezine \& Manzini: ABPEE.

Guimarães, T. G. \& Ourofino, V. T. A. T. (2007). Estratégias de identificação do aluno com altas habilidades/superdotação. Em D. S. Fleith (Org.), A construção de práticas educacionais para alunos com altas habilidades/superdotação. Brasília: Ministério da Educação, Secretaria de Educação Especial.

Ivo, M. C. (2012). A identificação de jovens com altas habilidades: uma abordagem winnicottiana da criatividade. Dissertação de Mestrado em Psicologia Clínica, Instituto de Psicologia, Universidade de São Paulo, São Paulo. Recuperado: 25 jun. 2015. Disponível: http://www.teses.usp.br/teses/disponiveis/47/47133/ tde-22082012-112113/.

Lei $n^{\circ}$ 9.394, de 20 de dezembro de 1996 (1996). Diretrizes e Bases da Educação Nacional. Brasília: Câmara dos Deputados.

Lima, D. M.M. P. (2011). O professor universitário frente às estratégias de identificação e atendimento ao aluno com altas habilidades/ 
superdotação. 2011. 127 f. Dissertação de Mestrado em Educação, Setor de Educação, Universidade Federal do Paraná, Curitiba. Recuperado: 02 jul. 2015. Disponível: http://www.ppge.ufpr.br/ teses/M11_Denise\%20Maria\%20de\%20Matos\%20Pereira\%20 Lima.pdf.

Marques, C. R. (2011). Levantamento de crianças com indicadores de altas habilidades em Jaboticabal/São Paulo. 2011. Dissertação de Mestrado em Educação Especial, Universidade Federal de São Carlos, São Carlos. Recuperado: 25 jun. 2015. Disponível: http:// www.bdtd.ufscar.br/htdocs/tedeSimplificado//tde_busca/arquivo. php?codArquivo=3848.

Marques, D. M. C. (2013). Reconhecimento por meio de indicadores da precocidade do aluno na educação infantil. Dissertação de Mestrado em Educação Especial, Universidade Federal de São Carlos, São Carlos. Recuperado: 25 jun. 2015. Disponível: http:// www.bdtd.ufscar.br/htdocs/tedeSimplificado//tde_arquivos/9/TDE2013-07-03T155950Z-5372/Publico/5232.pdf.

Martins, B. A. (2013). Alunos precoces com indicadores de altas habilidades/superdotação no ensino fundamental I: identificação e situações (des)favorecedoras em sala de aula. Dissertação de Mestrado em Educação, Faculdade de Filosofia e Ciências, Universidade Estadual Paulista, Marília. Recuperado: 02 jul. 2015. Disponível: http://www.marilia.unesp.br/Home/Pos-Graduacao/ Educacao/Dissertacoes/martins_ba_me_mar.pdf.

Martins, C. S. R. (2006). A identificação do aluno com potencial para altas habilidades/superdotação do sistema educacional adventista em Manaus. Dissertação de Mestrado em Educação, Universidade Federal do Amazonas, Manaus. Recuperado: 25 jun. 2015. Disponível: http://www.tede.ufam.edu.br/bitstream/tede/3231/1/ Claudia\%20Solange\%20Rossi\%20Martins.pdf.

Martins, A. C. S. \& Alencar, E. S. (2011). Características desejáveis em professores de alunos com altas habilidades/superdotação. Revista Educação Especial, 24 (39), 31-46.

Negrini, T. (2009). A escola de surdos e os alunos com altas habilidades/superdotação: uma problematização decorrente do processo de identificação das pessoas surdas. Dissertação de Mestrado em Educação, Universidade Federal de Santa Maria, Santa Maria. Recuperado: 25 jun. 2015. Disponível: http:// cascavel.ufsm.br/tede/tde_busca/arquivo.php?codArquivo=2727.

Oliveira, E. C. B. B. (2014). Identificação de crianças precoces com indicadores de altas habilidades/superdotação pelos familiares e suas expectativas. Dissertação de Mestrado em Educação, Faculdade de Filosofia e Ciências, Universidade Estadual Paulista,
Marília. Recuperado: 25 jun. 2015. Disponível: http://www.marilia. unesp.br/Home/Pos-Graduacao/Educacao/Dissertacoes/oliveira_ ecbb_me_mar.pdf.

Omote, S. (2014). Produção Acadêmica em Educação Especial. Em S. Omote, A. A. S. Oliveira, \& M. C. M. Chacon (Orgs.), Ciência e Conhecimento em Educação Especial (pp. 13-24). São Carlos: Marquezine \& Manzini: ABPEE.

Pereira, C. E. S. (2010). Identificação de estudantes talentosos: uma comparação entre as perspectivas de Renzulli e Güenther. Dissertação de Mestrado em Psicologia, Universidade Federal de Juiz de Fora, Juiz de Fora. Recuperado: 02 jul. 2015. Disponível: http://www.ufjf.br/ppgpsicologia/files/2010/01/Carlos-Eduardo-deSouza-Pereira1.pdf.

Pérez, S. G. P. B. \& Freitas, S. N.(2011). Encaminhamentos pedagógicos com alunos com Altas Habilidades/Superdotação na Educação Básica: o cenário brasileiro. Educar em Revista, n. 41, 109-124.

Ragni, R. A. (2012). Reconhecimento do talento em alunos com perdas auditivas do ensino básico. Tese de Doutorado em Educação Especial, Universidade Federal de São Carlos, São Carlos. Recuperado: 25 jun. 2015. Disponível: http://www. bdtd.ufscar.br/htdocs/tedeSimplificado//tde_busca/arquivo. php?codArquivo=5599

Resolução $n^{\circ}$ 2, de 11 de setembro de 2001 (2001). Diretrizes Nacionais para Educação especial na educação básica. Brasília: Conselho nacional de educação. Câmara de educação básica.

Souza, P. M. (2011). Identificação e caracterização dos alunos com deficiência, transtornos globais do desenvolvimento e altas habilidades/superdotação, matriculados nas classes comuns do ensino regular, na rede pública estadual, em município do interior paulista. Dissertação de Mestrado em Ciências, Faculdade de Filosofia, Ciências e Letras de Ribeirão Preto, Universidade de São Paulo, Ribeirão Preto. Recuperado: 25 jun. 2015. Disponível: file:///C:/Users/User/Downloads/DISSERTACAO.pdf.

Vieira, N. J. W. (2005). Viagem a "Mojave-ókil": a trajetória na identificação das altas habilidades/superdotação em crianças de quatro a seis anos. Tese de Doutorado em Educação, Faculdade de Educação, Universidade Federal do Rio Grande do Sul. Recuperado: 25 jun. 2015. Disponível: https://www.lume.ufrgs.br/ bitstream/handle/10183/6834/000491113.pdf?sequence=1 . 


\section{Sobre as autoras}

Bárbara Amaral Martins (barbara.amts@gmail.com)

Doutoranda em Educação pela Faculdade de Filosofia e Ciências. Universidade Estadual Paulista - Campus de Marília. Docente do curso de Pedagogia da Universidade Federal do Mato Grosso do Sul - Campus do Pantanal.

Ketilin Mayra Pedro (ketilinp@yahoo.com.br)

Doutoranda em Educação pela Faculdade de Filosofia e Ciências. Universidade Estadual Paulista - Campus de Marília com período sanduíche na Universidade de Barcelona.

Clarissa Marques Maria Ogeda (clarissaogeda@gmail.com)

Graduanda em Pedagogia pela Faculdade de Filosofia e Ciências. Universidade Estadual Paulista - Campus de Marília. 